\title{
Open versus closed vitrification system of human oocytes and embryos: a systematic review and meta-analysis of embryologic and clinical outcomes
}

Hongcai Cai ${ }^{1}$, Jean Damascene Niringiyumukiza ${ }^{1}$, Yamin Li ${ }^{1}$, Qiaohong Lai ${ }^{2}$, Yinzhao $\mathrm{Jia}^{3}$, Ping Su ${ }^{1 *}$ and Wenpei Xiang ${ }^{1 *}$

\begin{abstract}
Background: The objective of this study was to carry out a systematic review and meta-analysis of embryologic and clinical outcomes following open versus closed vitrification of human oocytes and embryos.

Methods: An electronic literature search was conducted in main electronic databases up to June 30, 2018 using the following key terms: 'oocyte', 'embryo', 'blastocyst', 'vitrification', 'cryopreservation', 'device', 'survival rate', 'pregnancy rate', etc. A meta-analysis was performed using a random effect model to estimate the value of risk ratios (RRs) and 95\% confidence interval (CI). Subgroup analyses and sensitivity analyses were carried out to further confirm the results.

Results: Twelve (Eight prospective and four retrospective) studies comparing open versus closed vitrification of human oocytes or embryos were included. For prospective studies on oocytes, no evidence for a significant difference in cryosurvival rate $(\mathrm{RR}=0.91,95 \% \mathrm{Cl}: 0.80-1.03, P=0.14 ; n=2048)$ or clinical pregnancy rate $(\mathrm{RR}=1.29$, $95 \%$ Cl: $0.80-2.06, P=0.30 ; n=150$ ) was observed. Additionally, there were no significant differences between the two methods concerning secondary endpoints included positive BHCG rate, implantation rate, miscarriage rate, ongoing pregnancy rate, live birth rate, cancellation rate, babies born per transferred blastocysts, or multiple birth rate $(P>0.05)$. The results of the retrospective studies were similar as the prospective studies.
\end{abstract}

Conclusions: It is still impossible to conclude that closed vitrification system could be a substitution for open system in human oocyte and embryo cryopreservation based on current evidence. Therefore, more well-designed prospective studies addressing these issues are still warranted.

Keywords: Vitrification, Cryopreservation, Oocyte, Blastocyst, Embryo, Meta-analysis

\section{Background}

Vitrification is one of the cryopreservation methods applied in the field of assisted reproductive technology (ART). With its high efficiency and consistency, vitrification is becoming the principal approach for cryopreservation of human oocytes and embryos, taking the place of traditional slow freezing in ART [1]. Several studies

\footnotetext{
* Correspondence: 438833975@qq.com; wpxiang2010@gmail.com ${ }^{1}$ Family Planning Research Institute/Center of Reproductive Medicine, Tongji Medical College, Huazhong University of Science and Technology, Wuhan 430030, Hubei, China

Full list of author information is available at the end of the article
}

have proved that vitrification/warming is superior to slow-freezing/ thawing with regard to embryologic and clinical outcomes for oocytes, cleavage-stage embryos, and blastocysts [2-4], which have been further supported by a recent published systematic review and meta-analysis [5]. Hence, vitrification is becoming an extensively used approach for cryopreservation in reproductive centres all over the world, as has been recommended by the American Society for Reproductive Medicine [6].

However, there are still concerns about the application and promotion of this new technique, particularly regarding biosafety issues. Depending on whether there is

(C) The Author(s). 2018 Open Access This article is distributed under the terms of the Creative Commons Attribution 4.0 International License (http://creativecommons.org/licenses/by/4.0/), which permits unrestricted use, distribution, and 
direct contact with the liquid nitrogen $\left(\mathrm{LN}_{2}\right)$, vitrification is commonly categorised into two types: 'open' and 'closed' system. While open vitrification reaches extreme high cooling rates due to direct contact with $\mathrm{LN}_{2}$, risks for potential cross-contamination and disease transmission mediated through $\mathrm{LN}_{2}$ increase when considering long-term cryopreservation. Alternatively, closed vitrification can avoid direct contact with $\mathrm{LN}_{2}$ by substituting the high concentration of cryoprotectant, thus influencing the efficiency of cooling [1]. As a consequence, relative studies have reported a decrease in cryosurvival rate which was attributed to closed vitrification $[7,8]$. Currently, concerns about the long-term effects of vitrification on large-scale oocytes and embryos when using high concentrations of cryoprotectant, which is regarded as cytotoxic, still exist. Viral cross-contamination between human oocytes and/or embryos in containers for cryostorage has not been identified so far $[9,10]$. However, environmental bacteria were identified in all the samples of $\mathrm{LN}_{2}$ collected from the oocyte/embryo containers, including open and closed devices, which could potentially do harm to the development of gametes and embryos [11]. Thus, it is still argued that the exposure of samples in open containers where environmental pathogens may exist can raise the contingent infectious danger for any IVF laboratory [12].

For the time being, however, results from different IVF laboratories around the world on the effects and safety of these two kinds of vitrification conflict. Regarding embryo cryopreservation, several studies reported comparable results between open and closed vitrification on survival rates after thawing [13-18]; while others demonstrated that closed vitrification outperformed the open one $[19,20]$. For oocytes vitrification, some suggested that 'open' was better than 'closed' in post-thawing survival rates $[7,8,21]$; others found no statistical differences between these two methods [22, 23]. In addition, the rest of the embryologic and clinical outcomes differed from each other according to the studies mentioned above. Therefore, our study aims to compare the embryological and reproductive outcomes between open and closed vitrification for human oocytes and embryos (cleavage-stage embryo and blastocyst), by searching for relevant literatures through electronic databases in the past decades, thus attempting to provide evidence-based medical support for clinically infertile couples seeking fertility cryopreservation.

\section{Methods}

\section{Search strategy}

Studies were searched for according to the date of June 30, 2018, through several main electronic databases, including PubMed, MEDLINE, EMBASE, Cochrane Central Register of Controlled Trials (CENTRAL), Cochrane
Libraries, World Health Organization (WHO) International Clinical Trials Registry Platform (ICTRP), Clinicaltrials.gov, and Current Controlled Trials to identify those which assessed embryological and reproductive outcomes by using oocytes and/or embryos vitrified by open or closed devices from women undergoing ART. The following search terms were applied, 'oocyte, 'embryo,' 'blastocyst,' 'cleavage-stage embryo', 'vitrification, 'cryopreservation', 'cryosurvival rate,' 'pregnancy rate', and 'live birth rate', without language restrictions. These searches were limited to human studies. Additionally, relevant studies from the citation list of all retrieved publications and review articles were hand-searched. We contacted the corresponding authors for further information if the primary studies were inadequate for analysis. Our study was approved by the Institutional Review Board of Family Planning Research Institute of Tongji Medical College, Huazhong University of Science and Technology.

\section{Study selection and eligibility criteria}

Two reviewers (HCC and JDN) performed an initial screening of all titles and abstracts independently. Studies were considered eligible if they (1) were prospective or retrospective studies, (2) compared open with closed vitrification as cryopreservation approaches, (3) used oocytes and/or embryos (cleavage and/or blastocyst stage) as participants, (4) used embryological (cyrosurvival rate as primary outcome, and fertilization rate, cleavage rate, good quality embryo rate or implantation rate, etc. as secondary outcomes) and/or reproductive outcomes (clinical pregnancy rate and/or live birth rate as primary outcome, and positive $\beta$ HCG rate, cancellation rate, miscarriage rate, ongoing pregnancy rate, or multiple birth rate, etc. as secondary outcomes.) as outcomes of interest (at least one primary indicator was involved in a single study), (5) were conducted on humans. Given that there were at least 30 different carrier tools published at present [1], for convenience, we here defined 'open' devices as those with direct contact between the sample-containing medium and $\mathrm{LN}_{2}$, such as Cryotop (KITAZATO BIOPHARMA CO., LTD, JAPAN), Cryoloop (Hampton Research CO., LTD, USA), open pulled straw, etc.; while 'closed' devices were those without direct contact with $\mathrm{LN}_{2}$, for instance, Cryotip (Irvine Scientific, USA), Rapid-i (Vitrolife, Sweden), vitrisafe (IVF Distribution $\mathrm{GmbH}$, Bregenz, Austria), closed pulled straw, and so on. Full texts were retrieved for further information if they satisfied the selection criteria. Studies conducted only on animals, without at least one primary indicator, and were not intact gametes or embryos although with elaborate design and of good quality, otherwise, were excluded. Review articles, conference abstracts, unpublished data, and cases reports were also considered as ineligible. Any discrepancy 
was resolved after hosting discussions with all authors until a consensus was reached.

\section{Data extraction and methodological quality evaluation}

A data extraction sheet was well designed beforehand to collect relevant information which included: demography (first author name, year, country, title of the study, journal, study period, number of patients/oocytes/embryos included, characteristics of the study participants and funding sources, conflicts of interest), methodology (study design, method of randomization, quality score), procedure (inclusion/exclusion criteria, ovarian stimulation and ovulation triggering protocols, type of cryo-carrier and cryoprotectant used, type of fertilization, stage at collection and transfer, cooling and warming rate, number of embryos transferred), and outcome data (relative embryological and reproductive outcome measures as previously described). Two independent authors extracted data and carefully assessed the quality of each study. In cases of disagreement, a consensus was reached after discussion between the two authors. To avoid inclusion of duplicate or overlapping samples, we meticulously compared the original areas of the studies. In the event of data overlapping, we included the latest version with the largest number of cases or the values of risk ratio (RR) and 95\% confidence intervals (CIs) that were adjusted. We used ROBINS-I tool for assessing the quality of all the included studies [24].

\section{Data synthesis and meta-analysis}

Meta-analyses of the included studies were employed to estimate the pooled RR value and $95 \%$ CIs of all the outcome measures. Heterogeneity of the studies was assessed using a $Q$ test and an $I^{2}$ index. A random effect model was used to estimate the value of risk ratios (RRs) and 95\% confidence interval (CI). Subgroup analyses were carried out according to the study location, study quality, and risks of bias, with the purpose of investigating substantial heterogeneity that might affect the cumulative evidence. When we detected substantial heterogeneity, a sensitivity analysis was then performed to explore possible explanations and further confirm the consistency of the outcomes. Statistical heterogeneity was taken into account when interpreting the results. An alpha value of 0.05 under a two-sided test was considered as statistically significant. The existence of publication bias was evaluated by establishing funnel plots as well as by performing Begg's [25] and Egger's test [26]. We used the Grading of Recommendations Assessment, Development and Evaluation (GRADE) system (study limitations, consistency of effect, imprecision, indirectness, and publication bias) to assess the quality of the evidence for all outcomes [27].

The meta-analysis and constructions of forest and funnel plots were performed with Review Manager Software (Version 5.3 for Mac; Copenhagen: The Nordic Cochrane
Centre, The Cochrane Collaboration, 2014). Stata/SE (version 12.0 for Mac) was utilised for Begg's and Egger's tests.

\section{Results}

Identification of literature

All studies comparing open versus closed vitrification of oocytes and/or embryos on embryologic and clinical reproductive outcomes were considered eligible in this systematic review and meta-analysis. The electronic search retrieved 397 records initially. Of these, 31 studies were excluded as duplicates and 366 records remained and were subsequently screened based on their title and abstract. Of these, 301 were consequently excluded because of obvious unrelated researches. The full texts of the remaining 65 articles were retrieved to be assessed for eligibility. Of these, 53 studies were excluded and the reasons for exclusion were as followed: not the type of comparison; lack of primary outcomes; animal research; review article. In total, 12 articles were included in the quantitative synthesis. The flow diagram of the selection procedure is presented in Fig. 1.

\section{Characteristics of the included studies and quality assessment}

There were 12 studies (eight prospective and four retrospective studies) included in quantitative analysis. These consisted of five studies on oocytes vitrification and nine studies on embryo vitrification, among which the data of two studies extracted from the oocytes studies [7, 22]. In total, 2577 oocytes (1330 for open and 1247 for closed vitrification, respectively) and 3640 embryos (2024 for open and 1616 for closed vitrification, respectively; data from one study [22] was not counted because the author didn't mention the number of embryos thawed in the study) were included for quantitative analysis. The language of the included studies was English, except for one in French [21]. The participants in these studies mainly came from Europe, North America, and East Asia. The basic characteristics of the selected literatures are listed on Table 1. Results of the methodological quality assessment showed that 3 of the prospective studies were of low risk of bias [8, 17, 22], 2 were moderate $[13,16]$ and 3 were of serious risk of bias $[20,21,23]$. The results of the quality assessment of the included retrospective studies were shown in Table 1.

\section{Publication bias evaluation}

Allowing for the difficulty of detecting and correcting for publication bias and other reporting biases, we tried to minimise their potential influence by ensuring a comprehensive search for eligible studies and by paying attention to the duplicated data. Results of Begg's and Egger's tests revealed that no significant publication biases were shown among the included studies, as presented in Tables 2 and 3. 


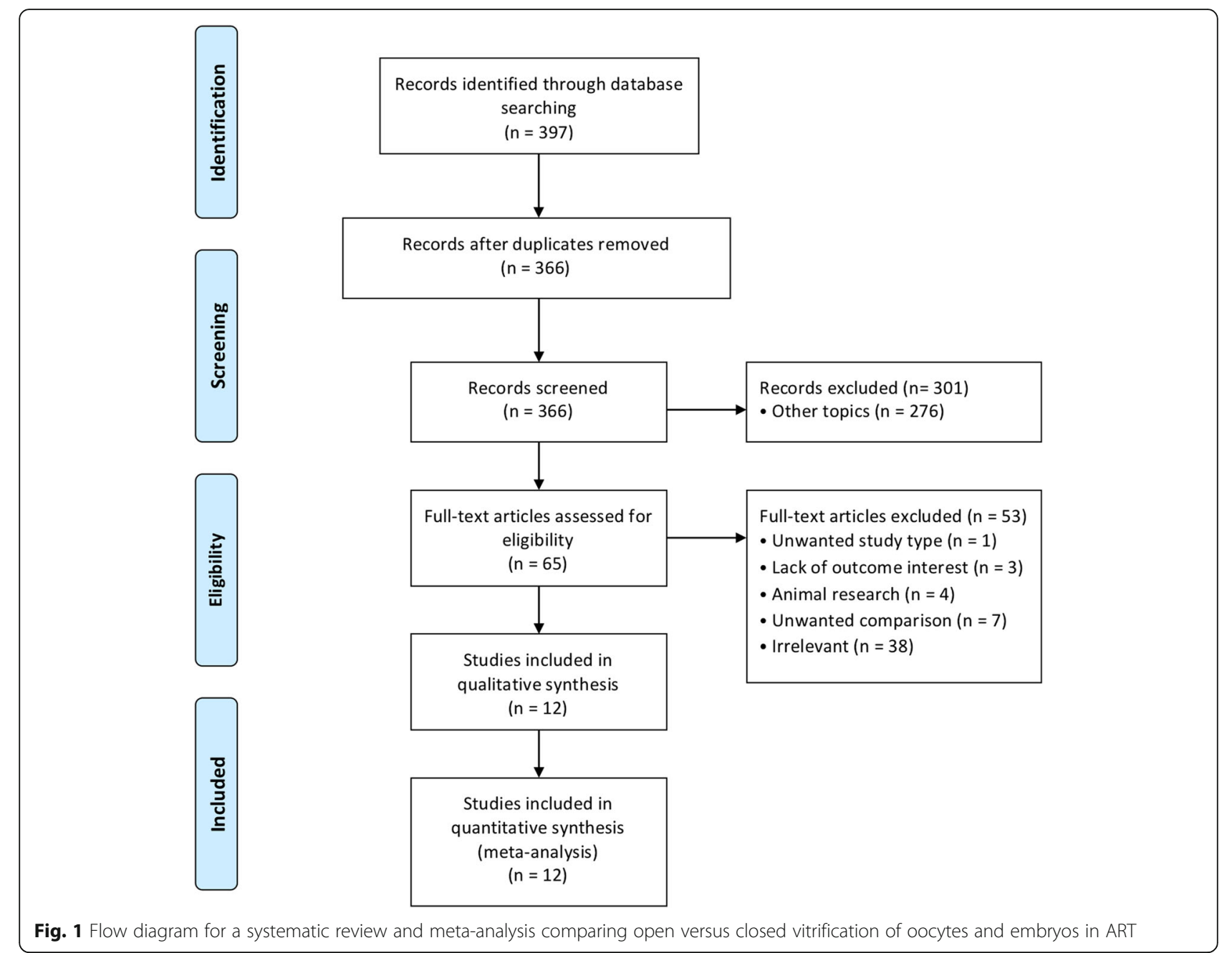

Open versus closed vitrification on oocytes: Embryologic and clinical outcomes

Five studies concerning oocytes vitrification were included for meta-analysis (See summary in Table 2). No evidence for a difference was found in regards to the outcome of the cryosurvival rate when comparing closed and open vitrification on oocytes, respectively $(\mathrm{RR}=0.91,95 \% \mathrm{CI}$ : $0.80-1.03, P=0.14 ; n=2048,986$ versus 1062 oocytes; $I^{2}$ $=91 \%$, low quality evidence). There was no significant difference regarding the clinical pregnancy rate $(R R=1.29$, 95\% CI: $0.80-2.06, P=0.30 ; n=150$, 75 versus 75 transferred cycles). Similarly, no differences were identified between the groups regarding fertilization rate $(R R=1.02$, 95\% CI: $0.83-1.24, P=0.88 ; n=1517,733$ versus 784 oocytes; $I^{2}=92 \%$, low quality evidence), good quality embryo rate $(\mathrm{RR}=0.97,95 \% \mathrm{CI}: 0.84-1.11, P=0.62$; $n=1127,561$ versus 566 embryos; $I^{2}=0 \%$, moderate quality evidence), positive $\beta \mathrm{HCG}$ rate $(\mathrm{RR}=1.09$, 95\% CI: $0.75-1.59, P=0.64 ; n=234,109$ versus 125 transferred cycles; $I^{2}=22 \%$, moderate quality evidence $)$ miscarriage rate $(\mathrm{RR}=0.59,95 \% \mathrm{CI}$ : $0.20-$
$1.79, P=0.35 ; n=77,38$ versus 39 clinical pregnancy cycles; $I^{2}=0 \%$, moderate quality evidence), ongoing pregnancy rate $(\mathrm{RR}=1.24,95 \% \mathrm{CI}: 0.81-1.89, P=0.32 ; \mathrm{n}=234$, 125 versus 109 transferred cycles; $I^{2}=0 \%$, moderate quality evidence), or live birth rate ( $\mathrm{RR}=1.50,95 \% \mathrm{CI}$ : $0.91-2.48$, $P=0.11 ; n=150,75$ versus 75 clinical pregnancy cycles). Sensitivity analyses were performed by excluding the study with the largest weight, smallest weight, or highest heterogeneity, and ended up with semblable conclusions.

\section{Open versus closed vitrification on embryos: Embryologic and clinical outcomes}

A total of nine studies (five prospective and four retrospective studies) on embryos vitrification were adopted for quantitative analysis (See summary in Table 3). Four prospective studies on embryo cryopreservation (closed versus open) demonstrated no difference in cryosurvival rate $(\mathrm{RR}=0.98,95 \% \mathrm{CI}: 0.96-1.01, P=0.29 ; n=1619$, 724 versus 895 oocytes; $I^{2}=0 \%$, moderate quality evidence) and findings were consistent with three retrospective studies $(\mathrm{RR}=1.00,95 \% \mathrm{CI}$ : 0.97-1.03, $P=0.88$; 


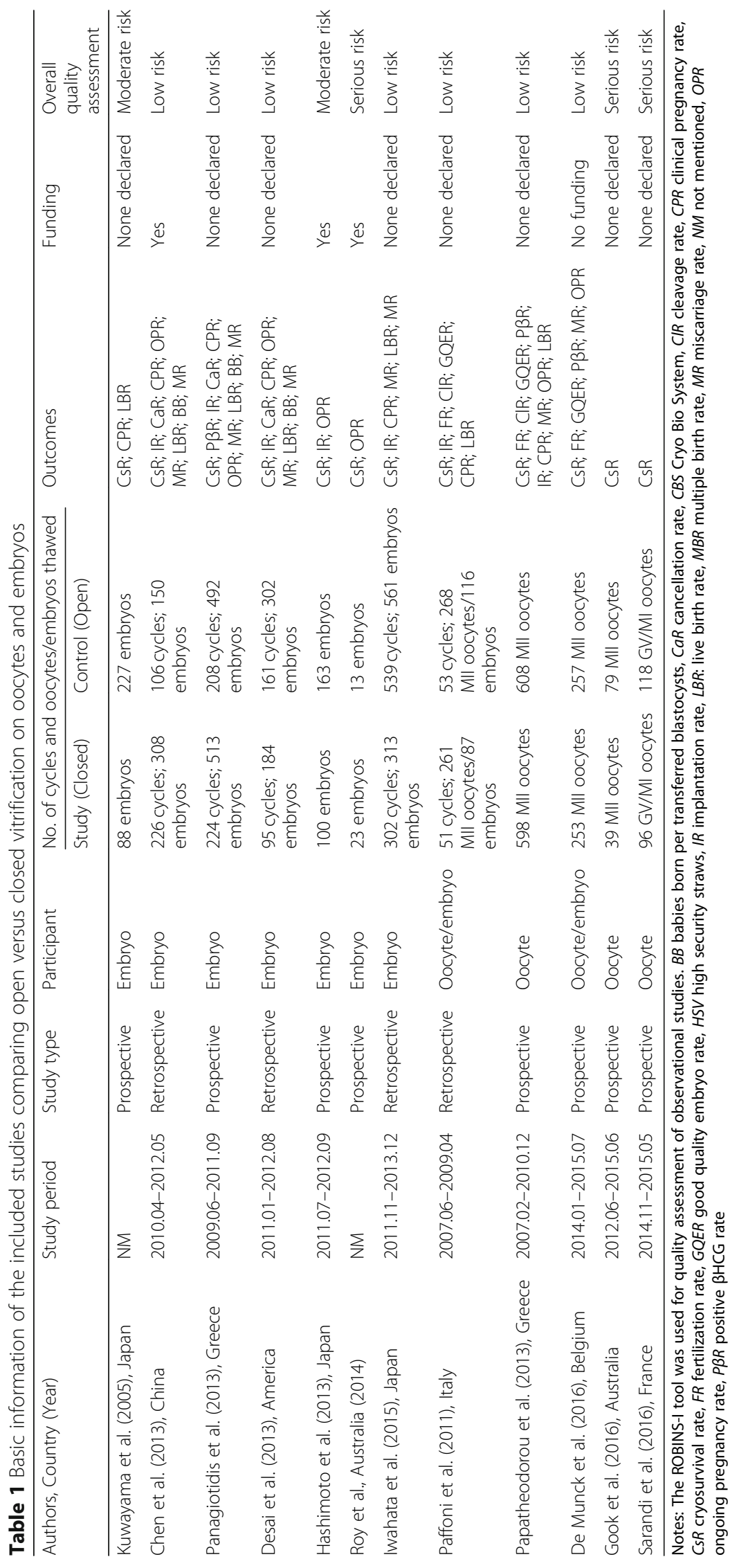




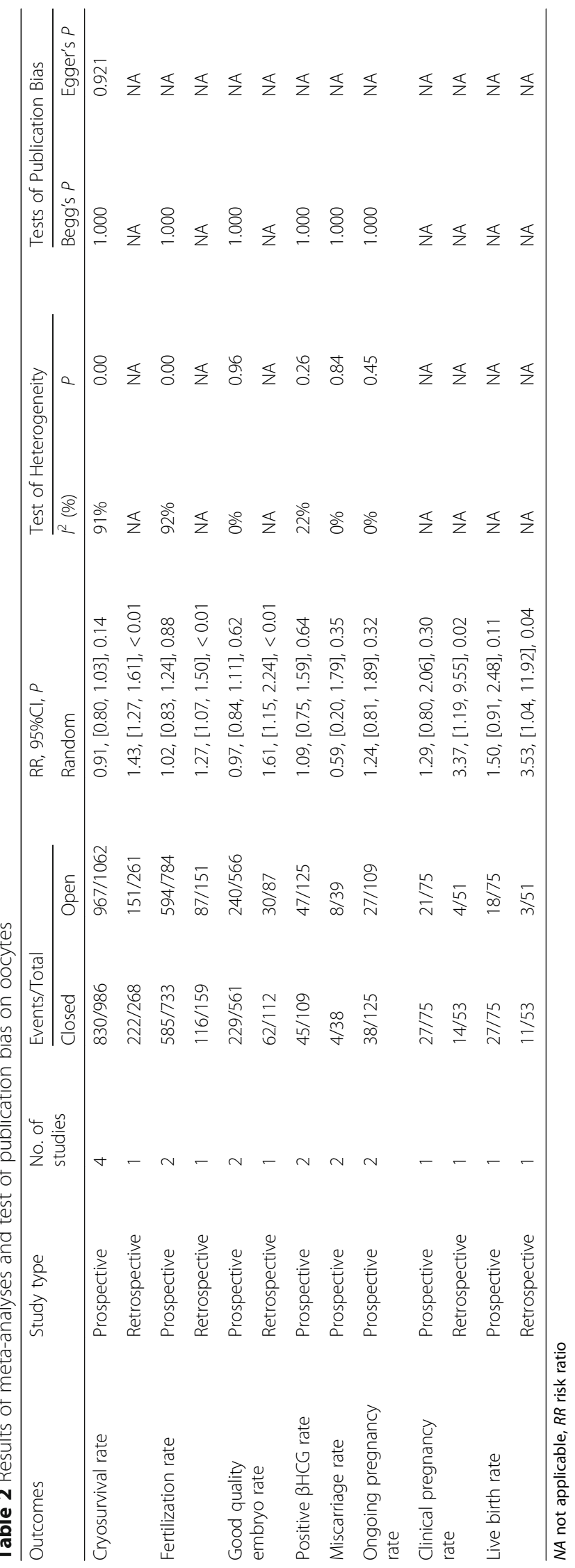




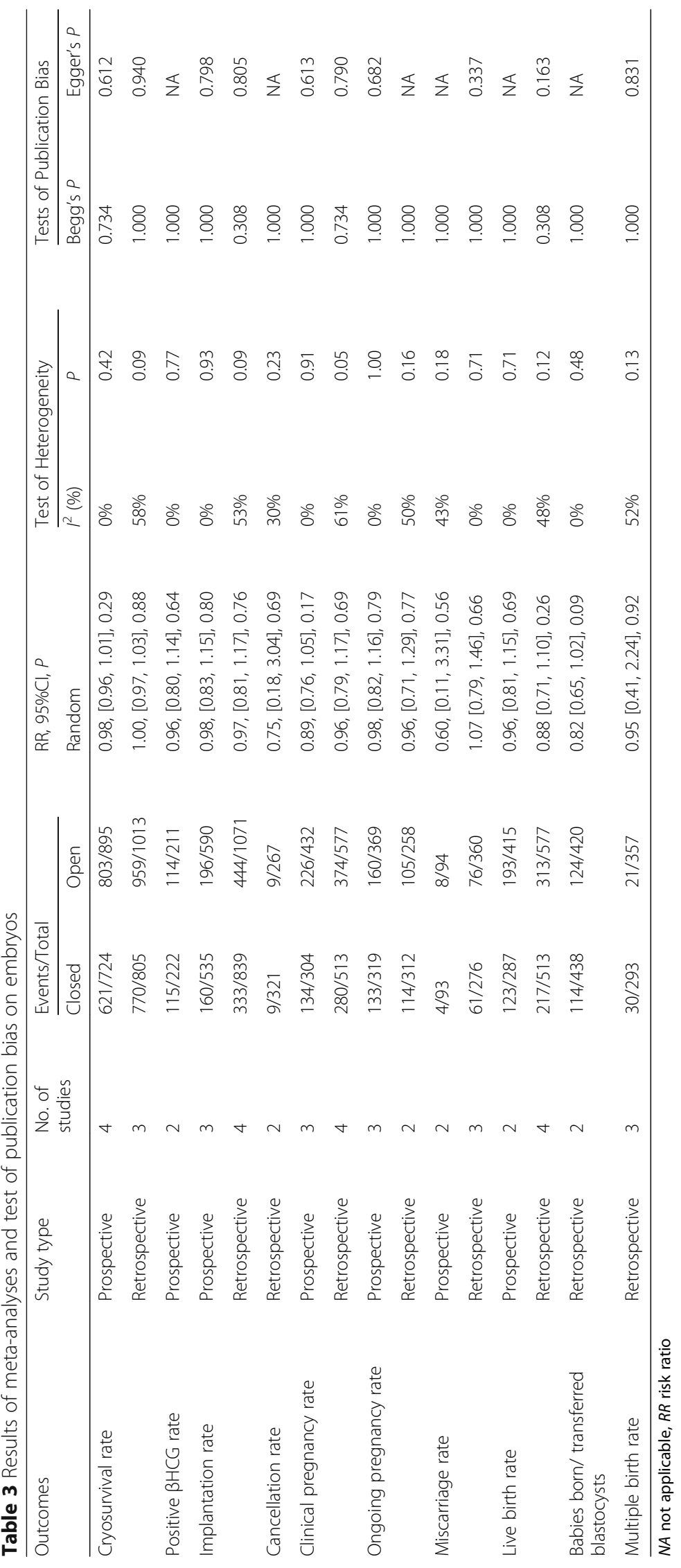


$n=1818,805$ versus 1013 oocytes; $\left.I^{2}=58 \%\right)$. There was no difference in clinical pregnancy rate $(\mathrm{RR}=0.89,95 \%$ CI: $0.76-1.05, P=0.17 ; n=736,304$ versus 432 transferred cycles; $I^{2}=0 \%$, moderate quality evidence), when comparing closed to open vitrification, respectively, which was also supported by four relevant retrospective studies (RR $=0.96,95 \% \mathrm{CI}$ : $0.79-1.17, P=0.69 ; n=1090$, 513 versus 577 oocytes; $I^{2}=58 \%$ ). Additionally, no evidence for differences in positive $\beta$ HCG rate $(R R=0.96$, 95\% CI: $0.80-1.14, P=0.63 ; n=433,222$ versus 211 transferred cycles; $I^{2}=0 \%$, moderate quality evidence), implantation rate $(\mathrm{RR}=0.97,95 \% \mathrm{CI}$ : $0.82-1.15, P=0.74$; $n=1125,535$ versus 590 transferred embryos; $I^{2}=0 \%$, moderate quality evidence), miscarriage rate $(R R=0.48$, 95\% CI: $0.15-1.54, P=0.22 ; n=187,93$ versus 94 clinical pregnancy cycles; $I^{2}=43 \%$, moderate quality evidence), ongoing pregnancy rate $(\mathrm{RR}=0.98,95 \% \mathrm{CI}$ : $0.82-1.16, P$ $=0.79 ; n=688,319$ versus 369 transferred cycles; $I^{2}=0 \%$, moderate quality evidence), live birth rate ( $R R=0.97,95 \%$ CI: $0.81-1.15, P=0.70 ; n=702,287$ versus 415 transferred cycles; $I^{2}=0 \%$, moderate quality evidence), cancellation rate $(\mathrm{RR}=0.75,95 \% \mathrm{CI}: 0.26-2.14, P=0.59 ; n=588,321$ versus 267 transferred cycles; $I^{2}=30 \%$, moderate quality evidence), babies born per transferred blastocysts $(\mathrm{RR}=0.82,95 \%$ CI: $0.66-1.03, P=0.08 ; n=858,438$ versus 420 transferred embryos; $I^{2}=0 \%$, moderate quality evidence), and multiple birth rate $(\mathrm{RR}=0.95,95 \% \mathrm{CI}$ : $0.41-2.24, P=0.92 ; n=650,293$ versus 357 live birth cycles; $I^{2}=52 \%$, moderate quality evidence) was shown. These findings were further confirmed by relevant retrospective studies and sensitivity analyses (Table 3 ).

\section{Subgroup analysis}

We planned the following subgroup analyses for the outcomes with high heterogeneity, such as cryosurvival rates, implantation rate, clinical pregnancy rate, and multiple birth rate, according to the differences of the original areas, study quality, and risks of bias, which also displayed no significant changes in related embryologic or clinical outcomes.

\section{Discussion}

In this systematic review and meta-analysis, we compared open versus closed vitrification for oocytes and embryos cryopreservation regarding post-thaw survival rate, clinical pregnancy rate, and other embryologic and clinical reproductive outcomes. We found that closed vitrification systems could achieve sound embryologic outcomes as 'open' ones for both human oocytes and embryos cryopreservation, although substantial heterogeneity existed in some of the outcomes. For more accurate findings, further studies should be performed to ensure sufficient data analysis.

Recently, two systematic reviews reported the reproductive outcomes of human oocytes and embryos comparing open versus closed vitrification $[28,29]$. One study suggested that it was not yet possible to conclude that closed vitrification is an aseptic alternative to open vitrification in human mature oocyte cryopreservation. In this study, only four articles concerning cyrosurvival rate after vitrification were included, of which the strength of evidence was low [29]. The other study regarding embryo vitrfication concluded with similar results on cryosurvival rates, implantation rates, clinical pregnancy rates and live birth rates, where seven articles were included. Although there was no significant difference between these two methods, the tendency of lower live birth rates with closed vitrification than with open vitrification could be clearly identified [28]. Our study was consistent with the previous conclusions, although low to moderate levels of evidence were achieved. This is probably due to the limited numbers of studies included and the variability in quality. Moreover, the high heterogeneity across the studies could be considered a limitation of the study.

Several factors, including the cooling and warming rates, the concentration of cryoprotectants, the solution volume, etc., should be taken into account when considering the effects of vitrification on human oocytes and embryos. Vitrification in cryobiology is essentially designed to eliminate ice formation in the medium containing the sample during the whole procedure (cooling, storage, and warming), which can be achieved by accelerating the cooling and warming rates, as well as increasing the concentration of cryoprotectants [30-32]. The high cooling and warming rates may help to alleviate chilling injury [33], while highly concentrated cryoprotectants may cause toxic and osmotic injury [34]. For this reason, the use of the smallest solution volume and the highest temperature conductivity between the sample-containing medium and the cooling or warming agent is required to achieve the highest cooling and warming rates [35]. Contrary to common beliefs, it has been proved that the intracellular concentration of cryoprotectant in vitrified embryos is lower than after slow freezing, although the solutions used in vitrification contain higher concentrations of cryoprotectants [36]. According to our results, the cooling rate of these closed systems was lower compared to open ones, whereas the warming rate was nearly the same as that of an open system. Therefore, outcome measures of closed systems with lower cooing rates may be more easily affected in some extent, particularly with respect to oocytes, mainly due to its small surface: volume ratio [37]. To some extent, this may partially explain the reason that the observed cryosurvival rates of oocytes in closed systems exhibited a downregulated trend than the open groups in most of the studies included, although the overall survival rates between both groups are not statistically different. Otherwise, such differences were more ambiguous among embryos after vitrification, with regard 
to embryologic and reproductive outcomes as presented in our systematic review.

Vitrification in cryobiology is essentially designed to eliminate ice formation in the medium containing the sample during the whole procedure (cooling, storage, and warming), which can be achieved by accelerating the cooling and warming rates, as well as increasing the concentration of cryoprotectants [30-32]. The high cooling and warming rates may help to alleviate chilling injury [33], while highly concentrated cryoprotectants may cause toxic and osmotic injury [34]. For this reason, the use of the smallest solution volume and the highest temperature conductivity between the sample-containing medium and the cooling or warming agent is required to achieve the highest cooling and warming rates [35]. Contrary to common beliefs, it has been proved that the intracellular concentration of cryoprotectant in vitrified embryos is lower than after slow freezing, although the solutions used in vitrification contain higher concentrations of cryoprotectants [36]. Due to the lack of data on the cooling and warming rates of vitrification included in this systematic review, subgroup analyses were unable to performed, so as to find out their effect on reproductive outcomes. Nonetheless, on the whole, the cooling rate of these closed systems was lower compared to open ones, whereas the warming rate was nearly the same as that of an open system. Therefore, outcome measures of closed systems with lower cooing rates may be more easily affected in some extent, particularly with respect to oocytes, mainly due to its small surface: volume ratio [37]. To some extent, this may partially explain the reason that the observed cryosurvival rates of oocytes in closed systems exhibited a downregulated trend than the open groups in most of the studies included, although the overall survival rates between both groups are not statistically different. Otherwise, such differences were more ambiguous among embryos after vitrification, with regard to embryologic and reproductive outcomes as presented in our systematic review.

Vitrification has been widely used for cryopreservation during the past decade by virtue of better outcomes when compared to traditional slow freezing. Nevertheless, closed vitrification, without the risk of biosafety, which also achieves sound embryological outcomes as open one does, should attract a lot of attention in the future. In this case, the efficiency and consistency of this method for mammalian oocytes and embryos could still be promoted by optimizing each step of the process, which could be widely applied to future clinical practice.

Drawbacks inherent to the quality and quantity of the included studies, in particular, some important outcomes such as clinical pregnancy rate, live birth rate on embryos, with only one study involving limited sample size incorporated into quantitative analysis, have weakened the strength of available evidence. In addition, few to no data are available for synthetic analysis on the safety aspects inherent to offspring generation derived from frozen-thawed gametes, given that convincing evidence is sparse, until recently a study reported that neonatal outcomes were not different after transfer of vitrified blastocysts compared with cleavage-stage embryos [38]. Another weakness in our systematic review was that we did not analyse studies that compared open or closed vitrified oocytes/embryos with fresh oocytes/embryos. Thus, more strong evidence is needed to better understand the real effect and safety that vitrification could achieve.

The optimal embryological and clinical outcomes of oocytes/embryos achieved with the use of vitrification over the last decade have important clinical implications, for instance, enhancing the cumulative live birth rate per oocyte retrieval cycle, extending time for embryo evaluation, enabling egg banking for donation and/or for oocyte accumulation, etc., which together allow a personalised approach in the care of different populations for medical or non-medical indications $[5,10,39,40]$. Nowadays, due to the avoidance of direct contact with $\mathrm{LN}_{2}$ and consequently, lowering the cross-contamination and disease transmission risks for long-term cryopreservation, closed vitrification has become more popular and widely used in IVF laboratories across the world [41].

\section{Conclusions}

In conclusion, closed vitrification system is still unable to be an aseptic alternative for open system when considering human oocyte and embryo cryopreservation based on current evidence. More large-scale studies with consolidated criteria and delicate design are needed to further evaluate the efficiency and biosafety of vitrification for human oocytes and embryos, especially focusing on the oocyte quality of older sub-infertile/infertile patients, as well as prolonging the length of follow-ups for offspring. Furthermore, it's necessary to keep on exploring novel cryoprotectant with low cytotoxicity and high efficiency, accelerating the cooling rate, and simplifying the procedure in all efforts to improve the technique of vitrification for ART. Given that the risk of biosafety still remains, otherwise, it is advocated that a rigorous process of standardization for vitrification should be placed on the agenda.

\footnotetext{
Abbreviations

ART: Assisted reproductive technology; Cl: Confidence interval; $L_{2}$ : Liquid nitrogen; RR: risk ratio

Acknowledgements

The authors acknowledge the assistance of Ms. Lian Hu, Ms. Ling Zhang and Ms. Xiaoyan Wan (Family planning research institute, Tongji Medical College, Huazhong University of Science and Technology) with the professional knowledge pertaining to cryopreservation techniques in IVF and Prof. Yuanzhong Zhou (School of Public Health, Zunyi Medical University) with statistical methodology and data analysis.
} 


\section{Funding}

The authors report no financial support.

\section{Availability of data and materials}

Please contact author for data requests.

\section{Authors' contributions}

Conception and design of the study (HCC, WPX); search strategy (HCC, JDN); data extraction, statistical analysis (HCC, JDN) and interpretation (all authors); drafting and revising the manuscript (HCC, YZJ); final approval of the manuscript to be published (all authors).

\section{Ethics approval and consent to participate}

Not applicable.

\section{Consent for publication}

Not applicable.

\section{Competing interests}

The authors declare that they have no competing interests.

\section{Publisher's Note}

Springer Nature remains neutral with regard to jurisdictional claims in published maps and institutional affiliations.

\section{Author details}

${ }^{1}$ Family Planning Research Institute/Center of Reproductive Medicine, Tongji Medical College, Huazhong University of Science and Technology, Wuhan 430030, Hubei, China. ${ }^{2}$ Center of Reproductive Medicine, Tongji Hospital, Tongji Medical College, Huazhong University of Science and Technology, Wuhan 430030, Hubei, China. ${ }^{3}$ Department of General Surgery, Union Hospital, Tongji Medical College, Huazhong University of Science and Technology, Wuhan 430030, Hubei, China.

Received: 5 July 2018 Accepted: 19 November 2018

Published online: 06 December 2018

\section{References}

1. Vajta G, Rienzi L, Ubaldi FM. Open versus closed systems for vitrification of human oocytes and embryos. Reprod BioMed Online. 2015;30:325-33.

2. Edgar DH, Gook DA. A critical appraisal of cryopreservation (slow cooling versus vitrification) of human oocytes and embryos. Hum Reprod Update. 2012;18:536-54.

3. Levi Setti PE, Porcu E, Patrizio P, Vigiliano V, de Luca R, d'Aloja P, et al. Human oocyte cryopreservation with slow freezing versus vitrification. Results from the National Italian Registry data, 2007-2011. Fertil Steril. 2014; 102:90-5.e2.

4. Li Z, Wang YA, Ledger W, Edgar DH, Sullivan EA. Clinical outcomes following cryopreservation of blastocysts by vitrification or slow freezing: a population-based cohort study. Hum Reprod. 2014;29:2794-801.

5. Rienzi L, Gracia C, Maggiulli R, LaBarbera AR, Kaser DJ, Ubaldi FM, et al. Oocyte, embryo and blastocyst cryopreservation in ART: systematic review and meta-analysis comparing slow-freezing versus vitrification to produce evidence for the development of global guidance. Hum Reprod Update. 2017;23:139-55.

6. (ASRM) ASfRM. Mature oocyte cryopreservation: a guideline. Fertil Steril. 2013;99:37-43.

7. Paffoni A, Guarneri C, Ferrari S, Restelli L, Nicolosi AE, Scarduelli $C$, et al. Effects of two vitrification protocols on the developmental potential of human mature oocytes. Reprod BioMed Online. 2011;22:292-8.

8. Papatheodorou A, Vanderzwalmen P, Panagiotidis Y, Prapas N, Zikopoulos K, Georgiou l, et al. Open versus closed oocyte vitrification system: a prospective randomized sibling-oocyte study. Reprod BioMed Online. 2013; 26:595-602.

9. Pomeroy KO, Harris S, Conaghan J, Papadakis M, Centola G, Basuray R, et al. Storage of cryopreserved reproductive tissues: evidence that cross-contamination of infectious agents is a negligible risk. Fertil Steril. 2010;94:1181-8.

10. Cobo A, Bellver J, de los Santos MJ, Remohi J. Viral screening of spent culture media and liquid nitrogen samples of oocytes and embryos from hepatitis B, hepatitis $C$, and human immunodeficiency virus chronically infected women undergoing in vitro fertilization cycles. Fertil Steril. 2012;97:74-8.

11. Molina I, Mari M, Martinez JV, Novella-Maestre E, Pellicer N, Peman J. Bacterial and fungal contamination risks in human oocyte and embryo cryopreservation: open versus closed vitrification systems. Fertil Steril. 2016; 106:127-32

12. Bielanski A, Vajta G. Risk of contamination of germplasm during cryopreservation and cryobanking in IVF units. Hum Reprod. 2009;24:2457-67.

13. Kuwayama M, Vajta G, leda S, Kato O. Comparison of open and closed methods for vitrification of human embryos and the elimination of potential contamination. Reprod BioMed Online. 2005;11:608-14.

14. Chen Y, Zheng X, Yan J, Qiao J, Liu P. Neonatal outcomes after the transfer of vitrified blastocysts: closed versus open vitrification system. Reprod Biol Endocrinol. 2013;11:107.

15. Desai NN, Goldberg JM, Austin C, Falcone T. The new rapid-i carrier is an effective system for human embryo vitrification at both the blastocyst and cleavage stage. Reprod Biol Endocrinol. 2013;11:41.

16. Hashimoto S, Amo A, Hama S, Ohsumi K, Nakaoka Y, Morimoto Y. A closed system supports the developmental competence of human embryos after vitrification : closed vitrification of human embryos. J Assist Reprod Genet. 2013;30:371-6.

17. Panagiotidis $Y$, Vanderzwalmen P, Prapas $Y$, Kasapi E, Goudakou M, Papatheodorou A, et al. Open versus closed vitrification of blastocysts from an oocyte-donation programme: a prospective randomized study. Reprod BioMed Online. 2013;26:470-6.

18. Iwahata $H$, Hashimoto $S$, Inoue $M$, Inoue $T$, Ito $K$, Nakaoka $Y$, et al. Neonatal outcomes after the implantation of human embryos vitrified using a closedsystem device. J Assist Reprod Genet. 2015;32:521-6.

19. Valbuena D, Poo ME, Aguilar-Gallardo C, Martinez S, Cobo AC, Pellicer A, et al. Comparison of Cryotip vs. Cryotop for mouse and human blastomere vitrification. Fertil Steril. 2012;97:209-17.

20. Roy TK, Brandi S, Tappe NM, Bradley CK, Vom E, Henderson C, et al. Embryo vitrification using a novel semi-automated closed system yields in vitro outcomes equivalent to the manual Cryotop method. Hum Reprod. 2014; 29:2431-8.

21. Sarandi S, Herbemont C, Sermondade N, Benoit A, Sonigo C, Poncelet C, et al. A prospective study to compare the efficiency of oocyte vitrification using closed or open devices. Gynecol Obstet Fertil. 2016:44:280-4.

22. De Munck N, Santos-Ribeiro S, Stoop D, Van de Velde H, Verheyen G. Open versus closed oocyte vitrification in an oocyte donation programme: a prospective randomized sibling oocyte study. Hum Reprod. 2016;31:377-84.

23. Gook DA, Choo B, Bourne H, Lewis K, Edgar DH. Closed vitrification of human oocytes and blastocysts: outcomes from a series of clinical cases. $J$ Assist Reprod Genet. 2016;33:1247-52.

24. Sterne JA, Hernan MA, Reeves BC, Savovic J, Berkman ND, Viswanathan M et al. ROBINS-I: a tool for assessing risk of bias in non-randomised studies of interventions. BMJ. 2016:355:i4919.

25. Begg CB, Mazumdar M. Operating characteristics of a rank correlation test for publication bias. Biometrics. 1994;50:1088-101.

26. Egger M, Davey Smith G, Schneider M, Minder C. Bias in meta-analysis detected by a simple, graphical test. BMJ. 1997;315:629-34.

27. Guyatt G, Oxman AD, Akl EA, Kunz R, Vist G, Brozek J, et al. GRADE guidelines: 1. Introduction-GRADE evidence profiles and summary of findings tables. J Clin Epidemiol. 2011;64:383-94.

28. Youm HS, Choi JR, Oh D, Rho YH. Closed versus open vitrification for human blastocyst cryopreservation: a meta-analysis. Cryobiology. 2017; 77:64-70.

29. Youm HS, Choi JR, Oh D, Rho YH. Survival rates in closed and open Vitrification for human mature oocyte cryopreservation: a meta-analysis. Gynecol Obstet Investig. 2018:83:268-74.

30. Hopkins JB, Badeau R, Warkentin M, Thorne RE. Effect of common cryoprotectants on critical warming rates and ice formation in aqueous solutions. Cryobiology. 2012;65:169-78.

31. Mukaida T, Oka C. Vitrification of oocytes, embryos and blastocysts. Best Pract Res Clin Obstet Gynaecol. 2012;26:789-803.

32. Mandawala AA, Harvey SC, Roy TK, Fowler KE. Cryopreservation of animal oocytes and embryos: current progress and future prospects. Theriogenology. 2016;86:1637-44.

33. Mazur P, Cole KW, Schreuders PD, Mahowald AP. Contributions of cooling and warming rate and developmental stage to the survival of Drosophila embryos cooled to -205 degrees C. Cryobiology. 1993;30:45-73. 
34. Vajta G, Holm P, Kuwayama M, Booth PJ, Jacobsen H, Greve T, et al. Open pulled straw (OPS) vitrification: a new way to reduce cryoinjuries of bovine ova and embryos. Mol Reprod Dev. 1998;51:53-8.

35. Yavin S, Arav A. Measurement of essential physical properties of vitrification solutions. Theriogenology. 2007;67:81-9.

36. Vanderzwalmen P, Connan D, Grobet L, Wirleitner B, Remy B,

Vanderzwalmen S, et al. Lower intracellular concentration of cryoprotectants after vitrification than after slow freezing despite exposure to higher concentration of cryoprotectant solutions. Hum Reprod. 2013;28:2101-10.

37. Sansinena M, Santos MV, Zaritzky N, Chirife J. Numerical simulation of cooling rates in vitrification systems used for oocyte cryopreservation. Cryobiology. 2011;63:32-7.

38. Belva F, Bonduelle M, Roelants M, Verheyen G, Van Landuyt L. Neonatal health including congenital malformation risk of 1072 children born after vitrified embryo transfer. Hum Reprod. 2016:31:1610-20.

39. Stoop D, Nekkebroeck J, Devroey P. A survey on the intentions and attitudes towards oocyte cryopreservation for non-medical reasons among women of reproductive age. Hum Reprod. 2011;26:655-61.

40. Fertility preservation and reproduction in patients facing gonadotoxic therapies: a committee opinion. Fertil Steril. 2013;100:1224-31.

41. Argyle CE, Harper JC, Davies MC. Oocyte cryopreservation: where are we now? Hum Reprod Update. 2016;22:440-9.

Ready to submit your research? Choose BMC and benefit from:

- fast, convenient online submission

- thorough peer review by experienced researchers in your field

- rapid publication on acceptance

- support for research data, including large and complex data types

- gold Open Access which fosters wider collaboration and increased citations

- maximum visibility for your research: over $100 \mathrm{M}$ website views per year

At BMC, research is always in progress.

Learn more biomedcentral.com/submissions 